\title{
Prediction of cavernous sinus invasion in patients with Cushing's disease by magnetic resonance imaging
}

\author{
*Panagiotis Mastorakos, MD, PhD,,2 Davis G. Taylor, MD, ${ }^{1}$ Ching-Jen Chen, MD, ${ }^{1}$ \\ Thomas Buell, MD, ${ }^{1}$ Joseph H. Donahue, MD, ${ }^{3}$ and John A. Jane Jr., MD'1 \\ 1Department of Neurological Surgery, University of Virginia Health Science Center, University of Virginia, Charlottesville, Virginia; \\ ${ }^{2}$ Department of Neurological Surgery, NIH/NINDS, Bethesda, Maryland; and ${ }^{3}$ Department of Radiology and Medical Imaging, \\ University of Virginia Health Science Center, University of Virginia, Charlottesville, Virginia
}

\begin{abstract}
OBJECTIVE Cavernous sinus invasion (CSI) in Cushing's disease (CD) negatively affects the probability of complete resection, biochemical cure, and need for adjuvant therapy. However, the prediction of CSI based on MRI findings has been inconsistent and variable. Among macroadenomas, the Knosp classification is the most widely utilized radiographic predictor of CSI, but its accuracy in predicting CSI and the probability of gross-total resection is limited in the setting of microadenomas or Knosp grade 0-2 macroadenomas. The authors noticed that the presence of a triangular shape of adenomas adjacent to the cavernous sinus on coronal MR images is frequently associated with CSI. The authors aimed to determine the correlation of this radiographic finding ("sail sign" [SS]) with CSI.
\end{abstract}

METHODS The authors performed a retrospective review of all patients with a pituitary lesion $<20 \mathrm{~mm}$ and a biochemical diagnosis of CD treated with endoscopic or microscopic transsphenoidal resection from November 2007 to May 2017. Overall 185 patients with CD were identified: 27 were excluded for negative preoperative imaging, 32 for lacking tumors adjacent to the sinus, 7 for Knosp grade 3 or higher, and 4 for inadequate intraoperative assessment of the CSI. Following application of inclusion and exclusion criteria, 115 cases were available for statistical analysis. Intraoperative CSI was prospectively evaluated at the time of surgery by one of two neurosurgical attending surgeons, and MRI data were evaluated retrospectively by a neurosurgical resident and attending neuroradiologist blinded to the intraoperative results.

RESULTS A positive SS was identified in 23 patients (20\%). Among patients with positive SS, $91 \%$ demonstrated CSI compared to $10 \%$ without an SS ( $<<0.001)$. Using the SS as a predictor of CSI provided a sensitivity of 0.7 and a specificity of 0.98 , with a positive predictive value (PPV) of 0.91 and a negative predictive value of 0.9 . Among patients with positive SS, $30 \%$ did not achieve immediate postoperative remission, compared to $3.3 \%$ of patients without an SS (p< 0.001).

CONCLUSIONS The presence of a positive SS among Cushing's adenomas adjacent to the CS provides strong PPV, specificity, and positive likelihood ratio for the prediction of CSI. This can be a useful tool for preoperative planning and for predicting the likelihood of long-term biochemical remission and the need for adjuvant radiosurgery.

https://thejns.org/doi/abs/10.3171/2018.2.JNS172704

KEYWORDS Cushing's disease; pituitary microadenoma; cavernous sinus invasion; retrospective study; sail sign; pituitary surgery

$\mathrm{C}$ AVERNOUS sinus invasion (CSI) in Cushing's disease (CD) is associated with reduced incidence of grosstotal resection and endocrine remission, increased morbidity, and need for adjuvant therapy.1,10,21 Invasion of the cavernous sinus (CS) by pituitary adenomas based on
MRI has been inconsistent and variable, with no single method demonstrating an ability to accurately predict CSI, even among macroadenomas (in which CSI is more common). ${ }^{4,7}$ Unlike other pituitary adenomas that typically present as macroadenomas, $\mathrm{CD}$ is more likely to present

ABBREVIATIONS ACTH = adrenocorticotropic hormone; $\mathrm{CD}=$ Cushing's disease; $\mathrm{CS}=$ cavernous sinus; $\mathrm{CSI}=\mathrm{CS}$ invasion; $\mathrm{NPV}=$ negative predictive value; $\mathrm{PPV}=$ positive predictive value; $S S$ = sail sign; VIBE = volumetric interpolated breath-hold examination.

SUBMITTED October 26, 2017. ACCEPTED February 8, 2018.

INCLUDE WHEN CITING Published online July 6, 2018; DOI: 10.3171/2018.2.JNS172704.

${ }^{*}$ P.M. and D.G.T. contributed equally to this work. 

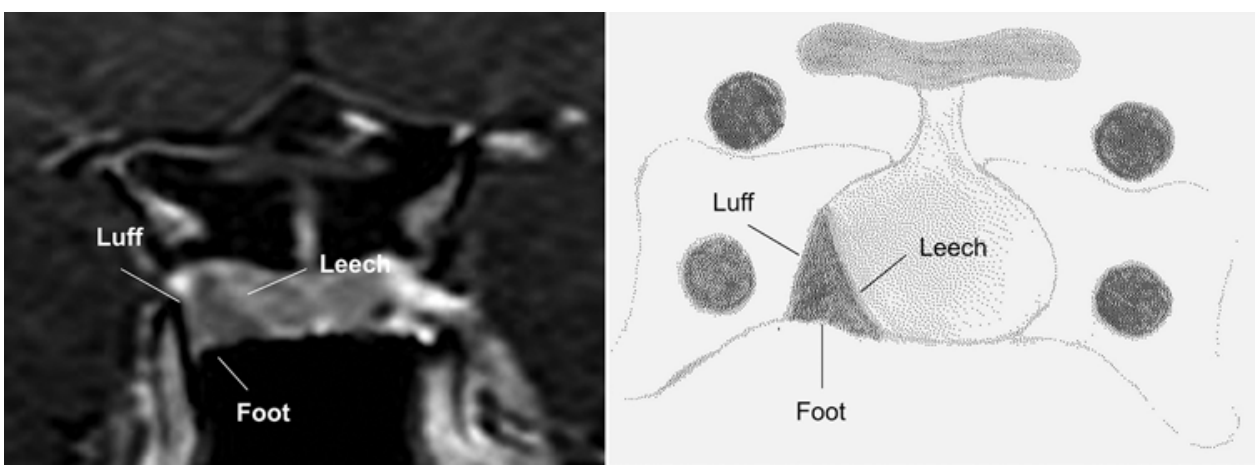

FIG. 1. Example and illustration of the sail sign. Particular attention should be given to the strictly triangular appearance of this imaging finding. Note the sail-like appearance of the finding with corresponding leech, luff, and foot.

while the tumor is still quite small. Despite their small size, a significant number of $\mathrm{CD}$ adenomas are found to have CSI at surgery, and no existing radiographic grading system reliably predicts this finding. ${ }^{15}$ We noticed that in some $\mathrm{CD}$ adenomas adjacent to the CS there is a particular triangular shape demonstrated on coronal MR images that frequently predicts CSI. This distinct triangular shape with sharp borders is akin to the appearance of a ship's sail (the leech, luff, and foot) (Figs. 1 and 2 upper), which deviates from the standard, well-circumscribed, and circular or ellipsoid shape more commonly appreciated (Fig. 2 lower). We therefore conducted a cohort study of retrospectively reviewed preoperative images and prospectively collected operative data to determine the correlation between this radiographic finding and CSI as seen at surgery.

\section{Methods}

\section{Patient Population}

Following institutional review board approval, we performed a retrospective evaluation of patients with pituitary lesions $<20 \mathrm{~mm}$ and with a biochemical diagnosis and histopathological confirmation of CD who underwent endoscopic or microscopic transsphenoidal resection by
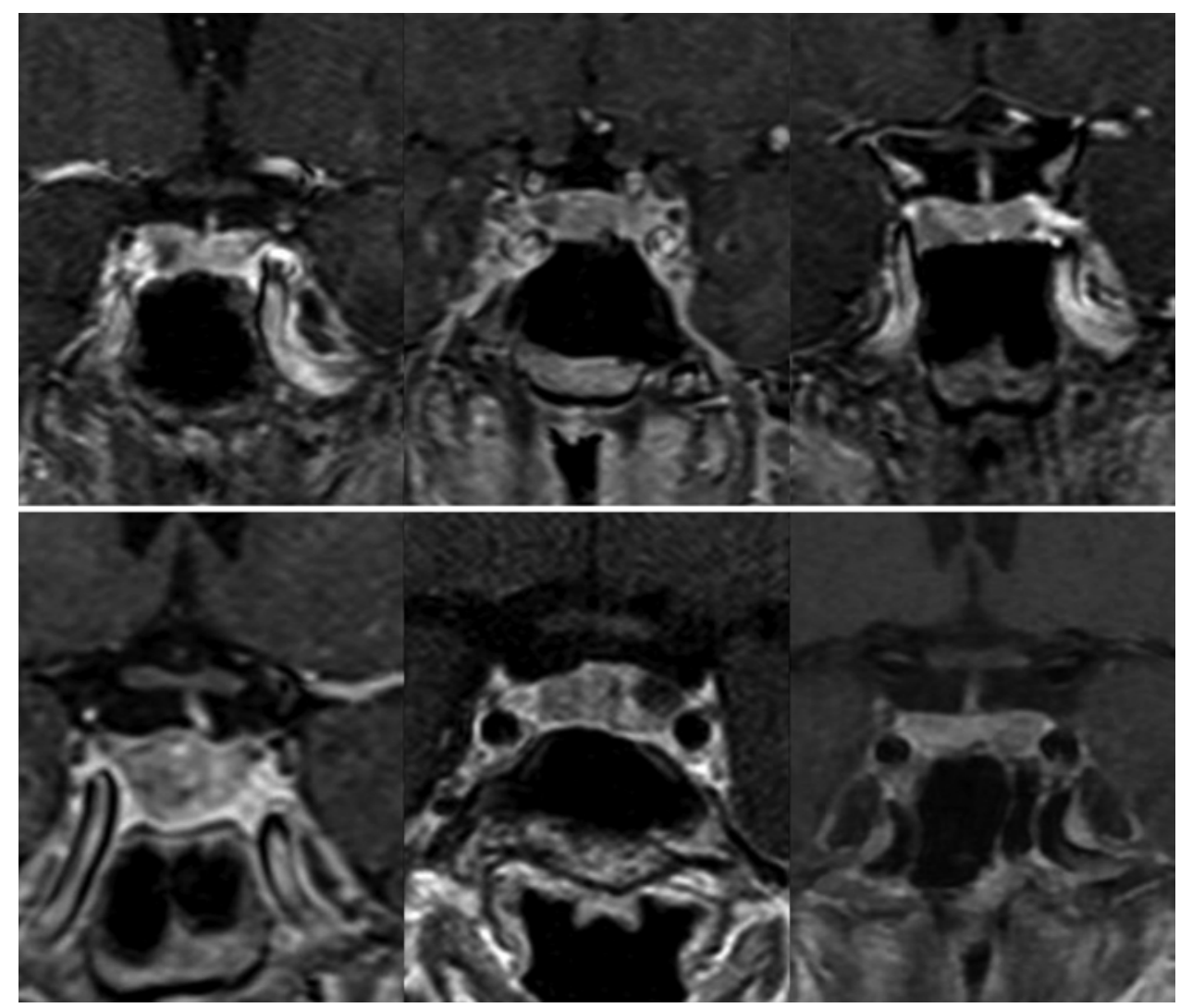

FIG. 2. Upper: Representative T1-weighted postcontrast VIBE images demonstrating the presence of the SS. Lower: Representative T1-weighted postcontrast VIBE images demonstrating typical ellipsoid adenomas adjacent to the CS (negative SS). 
TABLE 1. Patient population characteristics

\begin{tabular}{lc}
\hline \multicolumn{1}{c}{ Characteristic } & Value $(\%)$ \\
\hline Patients & 115 \\
\hline Female & $91(79 \%)$ \\
\hline Mean age $(\mathrm{yrs})$ & 39.4 \\
\hline Mean diameter of tumor $(\mathrm{mm})$ & 7.8 \\
\hline Microadenomas & $84(73 \%)$ \\
\hline Prevalence of CSI & $30(26 \%)$ \\
\hline Postop remission* & $105(91.3 \%)$ \\
\hline Adjuvant radiosurgery & $16(13.9 \%)$ \\
\hline
\end{tabular}

Values are presented as the number of patients (\%) unless otherwise indicated.

${ }^{*}$ Cortisol $<1 \mu \mathrm{g} / \mathrm{dl}$ prior to discharge.

either of two senior neurosurgeons (Edward H. Oldfield and John A. Jane Jr.) from November 2007 to May 2017. Inclusion criteria required available preoperative postcontrast T1-weighted MR images (185 patients). Patients were excluded if they had negative preoperative imaging (27 patients), if the tumor was not adjacent to the CS (32 patients), if the lesion were a Knosp grade of 3 or greater (7 patients), or if CSI could not be assessed intraoperatively or if this was not mentioned in the prospective database (4 patients). As a result, 115 patients were available for statistical analysis.

\section{Operative Findings}

CSI was determined by the senior neurosurgeons at the time of surgery based upon evidence of gross invasion of the tumor into the CS wall, free tumor within the CS, or both. Findings were entered into a prospective database shortly after surgery by the senior neurosurgeons.

\section{Radiographic Analysis}

All patients underwent routine preoperative MRI prior to surgery. Coronal T1-weighted postcontrast spin echo images, postcontrast volumetric interpolated breath-hold examination (VIBE) images, and T2-weighted images were evaluated retrospectively by one of the authors (P.M.), who was blinded to the surgical findings, and the images were classified accordingly based on the presence (Fig. 2 upper) or absence (Fig. 2 lower) of the so-called sail sign (SS). To evaluate interobserver reliability, the images were independently evaluated by an attending neuroradiologist (J.H.D.), who was also blinded to the surgical findings.

\section{Statistical Analysis}

The chi-square test was used to examine statistical differences of qualitative data. We proceeded to evaluate the SS as a predictor of CSI by sensitivity, specificity, positive predictive value (PPV), negative predictive value (NPV), positive likelihood ratio, and negative likelihood ratio.

\section{Results \\ Demographics}

Among the 115 patients whose data were eligible for analysis, $79 \%$ were female and $21 \%$ were male. The aver-
TABLE 2. Sail sign and correlation with CSI pattern

\begin{tabular}{lc}
\hline \multicolumn{1}{c}{ Variable } & Value $^{*}$ \\
\hline Prevalence of SS & $23(20 \%)$ \\
\hline CSI for +SS/-SS & $91 \% / 10 \%(p<0.001)$ \\
\hline+ SS for +CSI/-CSI & $70 \% / 2.4 \%(p<0.001)$ \\
\hline Sensitivity/specificity & $0.7 / 0.98$ \\
\hline PPV/NPV & $0.91 / 0.90$ \\
\hline LR+/LR- & $29.8 / 0.31$ \\
\hline CS wall invasion for +SS/-SS & $80 \% / 33 \%(p=0.014)$ \\
\hline Postop remission for +SS/-SS & $70 \% / 96.7 \%(p<0.001)$ \\
\hline
\end{tabular}

$\mathrm{LR}+=$ positive likelihood ratio; $\mathrm{LR}-=$ negative likelihood ratio; +SS = positive SS; - SS = negative SS .

${ }^{*} p$ value calculated using chi-square test.

age age was 39.4 years. Of the 115 tumors, $84(73 \%)$ were microadenomas $(<10 \mathrm{~mm})$ and $31(26.9 \%)$ were macroadenomas (>10 mm). The average diameter of the adenomas was $7.8 \mathrm{~mm}$. A microscopic sublabial transsphenoidal approach was performed in 107 patients by a senior neurosurgeon (Edward H. Oldfield) and an endoscopic endonasal transsphenoidal approach was performed in 8 patients by the senior author (John A. Jane Jr.). CSI at surgery was seen in $26 \%$ of patients. A sample of the CS dura was obtained in 21 of the cases in which CSI was identified intraoperatively. Among these cases, 16 had CSI confirmed histologically. Remission, determined as a postoperative drop in the serum cortisol level to $1 \mu \mathrm{g} / \mathrm{dl}$ or less prior to discharge, was achieved in $91.3 \%$ of the patients. Demographic data are provided in Table 1.

\section{SS and CSI Among Adenomas $<20 \mathrm{~mm}$ in Diameter}

Retrospective blinded evaluation of preoperative images demonstrated a positive SS in 23 patients (20\%). Among patients with a positive SS, 91\% demonstrated CSI compared to $10 \%$ without an SS ( $p<0.001)$. Among patients with CSI, $70 \%$ had a positive SS compared to $2.4 \%$ of patients without CSI ( $\mathrm{p}<0.001)$. Using the SS as a predictor of CSI provided a sensitivity of 0.7 and a specificity of 0.98, with a PPV of 0.91 and an NPV of 0.9. Based on the likelihood ratio, the presence of an SS increases the probability of CSI by 29.8 and the lack of an SS decreased the probability of CSI by 3.2 (Table 2 ).

The size of the adenomas did not differ significantly between the two populations, with average diameters of $6.13 \mathrm{~mm}$ and $6.08 \mathrm{~mm}$ for adenomas with and without presence of SS, respectively. Among the 31 macroadenomas, 8 were Knosp grade 0, and these had no CSI and no SS present; 18 were Knosp grade 1, and of these, 4 had a positive SS and 8 had CSI intraoperatively; 5 were Knosp grade 2, and of these, 2 had a positive SS and 3 had CSI intraoperatively. Of note, among these patients all SS-positive adenomas had CSI intraoperatively.

\section{SS and CSI Among Microadenomas}

We then evaluated the accuracy of the SS among the 84 microadenomas, exclusively. Among patients with microadenomas, $20 \%$ had positive SS, and $85 \%$ of patients with a positive SS demonstrated CSI compared to $4.6 \%$ 
TABLE 3. Sail sign and correlation with CSI pattern for microadenomas

\begin{tabular}{lc}
\hline \multicolumn{1}{c}{ Variable } & Value $^{*}$ \\
\hline Prevalence of SS & $20(23.8 \%)$ \\
\hline CSI for +SS/-SS & $85 \% / 4.6 \%(p<0.001)$ \\
\hline +SS for +CSI/-CSI & $85 \% / 4.6 \%(p<0.001)$ \\
\hline Sensitivity/specificity & $0.85 / 0.95$ \\
\hline PPV/NPV & $0.85 / 0.95$ \\
\hline LR+/LR- & $18.1 / 0.16$ \\
\hline CS wall invasion for +SS/-SS & $87 \% / 40 \%(p=0.007)$ \\
\hline Postop remission for +SS/-SS & $70 \% / 98.4 \%(p<0.001)$ \\
\hline
\end{tabular}

* $p$ value calculated using the chi-square test.

of patients without an SS $(\mathrm{p}<0.001)$. For patients with microadenomas, the SS provided sensitivity of 0.85 and specificity of 0.95 . Based on likelihood ratio, the presence of an SS increased the probability of CSI 18.1 times and the absence of an SS decreased the probability of CSI 6.4 times (Table 3).

\section{Interobserver Variability}

The interobserver variability was evaluated between two independent, blinded raters. The overall agreement was $87 \%$, with a free marginal Kappa value of 0.72 . The second rater had a sensitivity of 0.6 and a specificity of 0.96 , with a PPV of 0.86 and an NPV of 0.9 .

\section{SS and Invasion of CS Wall}

Among the population with surgically appreciated CSI and a positive SS, $80 \%$ had CS wall invasion and $20 \%$ were only found to have free tumor within the CS without evidence of invasion of the sinus wall. In contrast, among the population with known CSI and a negative SS, 33\% had CS wall invasion only compared with $67 \%$ with free tumor within the CS ( $\mathrm{p}=0.014$; Table 2$)$.

\section{Postoperative Course}

Following transsphenoidal surgery, 105 (91.3\%) of patients achieved remission prior to discharge. Among patients with surgically appreciated CSI, $26.6 \%$ did not achieve postoperative remission, compared to $2.4 \%$ of patients without CSI $(\mathrm{p}<0.001)$. For patients with a positive SS, $30 \%$ did not achieve postoperative remission, compared to $3.3 \%$ of patients without an SS ( $p<0.001$; Table 2). Similarly for microadenomas, $30 \%$ of patients with a positive SS did not achieve postoperative remission, compared to $1.6 \%$ of patients without an SS ( $p<0.001$; Table $3)$. Of the 10 patients who did not achieve immediate postoperative remission, 7 achieved remission following postoperative radiosurgery.

Adjuvant radiosurgery was pursued in 16 patients (13.9\%). Among patients with surgically appreciated CSI, $46.7 \%$ received adjuvant radiosurgery compared to $2.4 \%$ of patients without CSI $(\mathrm{p}<0.001)$. For patients with a positive SS, $43.5 \%$ underwent postoperative radiosurgery in contrast to $6.5 \%$ of patients without an SS (p < 0.001).

There were 3 documented recurrences. One of these patients had documented CSI at surgery and a positive SS, whereas the other 2 had not demonstrated CSI and did not have an SS.

\section{Discussion}

Cavernous sinus invasion by pituitary adenomas is associated with persistent endocrinopathy following surgery due to microscopic remnants of tumor cells, which, owing to the potent systemic effects of adrenocorticotropic hormone (ACTH) production, can lead to early symptom recurrence despite minimal residual tumor. ${ }^{8}$ When present, residual tumor may require either repeat surgery or adjuvant radiotherapy, which can take years to produce biochemical remission. ${ }^{18}$ As such, preoperative recognition of CSI is of utmost importance in both surgical planning and discussion with patients regarding expectations following surgery and need for adjuvant treatment.

To improve preoperative planning, multiple imaging classifications of pituitary adenomas have been developed to increase the likelihood of accurately identifying CSI. ${ }^{4,7}$ The Knosp classification for macroadenomas is the most widely utilized system and has been proven to correlate with the likelihood of achieving a gross-total resection. ${ }^{6,11,14}$ A pooled meta-analysis demonstrated that the radiographic inferolateral venous compartment obliteration and Knosp grade 3-4 tumors have the highest correlation with intraoperative CSI and the lowest correlation with gross-total resection. ${ }^{7}$ Knosp grade 0-2 lesions are generally considered resectable, but among these tumors, the Knosp classification does not offer accurate prediction of resection rates. ${ }^{5}$ Moreover, the Knosp classification system and other widely known systems, such as the Hardy classification system, were designed for interpretation of macroadenomas, and thus their application in the interpretation of CSI among microadenomas is limited. ${ }^{11,14}$

Image interpretation of microadenomas is particularly problematic in patients with CD. Up to $80 \%$ of patients with $\mathrm{CD}$ will present with a microadenoma, and the high incidence of incidentalomas in the general population has led to a false-negative and false-positive rate as high as $23 \%$ and $11.5 \%$ on MRI, respectively. ${ }^{3,12,17}$ Furthermore, there is no established imaging approach for microadenomas that reliably predicts CSI. Based on traditional interpretation of adenomas, Dickerman and Oldfield found that among 42 cases of recurrent CD due to CSI as seen at surgery, not a single patient had imaging characteristics to support this finding, and Lonser et al. demonstrated that accurate prediction of CSI occurred in only $22 \%$ of patients. ${ }^{8,15}$

Although there exists a typical central topological arrangement of $\mathrm{ACTH}$-producing pituicytes within the normal gland, laterally placed ACTH-producing tumors are frequently encountered. ${ }^{2,8,13}$ Laterally positioned Cushing's adenomas confer a greater risk of CSI due to disruption of the pseudocapsule dissection plane, which results in reduced likelihood of complete resection and a greater incidence of recurrent disease..$^{8,16,19,21}$ As reported here, we identified an imaging sign that predicts CSI with high specificity for pituitary adenomas adjacent to the CS. The SS denotes distinctive triangular margins of an adenoma in direct contact with the CS wall. This can be identified 
on coronal T1-weighted postcontrast spin echo images with 2- to 3-mm slice thickness, and, in cases in which tumor margins are indistinct on postcontrast imaging, an SS can still be appreciated on the dynamic postcontrast images or T2-weighted images. It must be stressed that regardless of which MRI sequence best depicts the lesion's contours, the luff of the SS must be produced by the adenoma directly contacting the CS wall, creating a linear lateral tumor margin. Following our analysis, the presence of the SS demonstrated a significant association with CSI compared to the absence of the SS $(91 \%$ vs $10 \%$, p < 0.001). Moreover, the specificity of 0.98 and PPV of 0.91 indicating the utility in accurately identifying those patients who are likely to have CSI are considerably greater than those appreciated by Lonser et al. when using traditional techniques and, therefore, should be recognized and appreciated by pituitary surgeons. . $^{5,15}$

Preoperative recognition of CSI is essential to discussions with patients regarding expectations of biochemical cure or the need for adjuvant therapy. ${ }^{16,20}$ Although surgical resection remains the treatment of choice for ACTHproducing tumors, radiosurgery remains the preferred adjuvant therapy for tumors with CSI or those that were incompletely resected. The presence of a positive SS on preoperative imaging (as determined by two independent and blinded reviewers) correlated well not only with CSI at surgery but also with failure to achieve remission immediately following surgery and therefore strongly correlated with the likelihood of adjuvant radiosurgery.

Interestingly, we found that a positive SS appears to be more predictive of invasion into the CS wall alone rather than freely into the CS proper (84\% vs 33\%, p < 0.05$)$. A report by Fernandez-Miranda et al. suggested preferential CSI of adenomas into CS compartments, which may explain the particular shape appreciated in our report. ${ }^{9}$ Based on this CS compartmentalization, we suspect that adenoma growth occurs within the CS wall of the posterior or inferior compartment, which continues to apply some degree of tension against the tumor margins to maintain the well-defined imaging findings of the leech, luff, and foot of the sail and thus creating the particular triangular shape appreciated in this finding.

We must acknowledge that though the NPV is quite good (0.9), it still allows a $10 \%$ chance of invasion and thus CSI cannot be ruled out entirely based on the presence or absence of this finding. As such, physicians should still discuss the potential of CS or dural invasion in adenomas adjacent to the CS regardless of the presence or absence of the SS.

In our cohort the interobserver agreement was $87 \%$ between a neurosurgical resident and an attending neuroradiologist. The agreement between the two observers was quite high despite relative inexperience with the rating system prior to evaluation and thus should be expected to improve with time. We should emphasize that particular attention must be given to the sharp triangular appearance herein demonstrated.

\section{Limitations}

As a retrospective study, there is potential for bias. Reviewers were blinded to the surgical findings to remove any potential bias of imaging interpretation. Also, the definition of CSI during surgery is surgeon dependent. However, the SS was predictive of histologically confirmed CSI with sensitivity 0.7 and specificity 0.95 . Nevertheless, given the significant specificity and PPV offered by the presence of the SS among laterally situated CD adenomas, pituitary surgeons may find this sign useful in treating patients with CD.

\section{Conclusions}

The presence of a positive SS among laterally situated CD adenomas has a strong PPV, specificity, and positive likelihood ratio of CSI. Lack of an SS does not rule out CSI. Appreciation of this finding and its correlation with CSI and postoperative remission can help surgeons in preoperative planning as well as in discussions with patients regarding the likelihood of achieving a long-term biochemical remission through surgery and the potential recommendation for adjuvant postoperative radiosurgery.

\section{Acknowledgments}

We would like to express our deepest gratitude to Dr. Edward H. Oldfield, who was involved with the development of the concept and the design of the study, and was the primary surgeon for the majority of patients included. We thank him for his contributions to this work and the neurosurgical literature, and the impact he has had in our personal lives.

\section{References}

1. Ahmadi J, North CM, Segall HD, Zee CS, Weiss MH: Cavernous sinus invasion by pituitary adenomas. AJR Am J Roentgenol 146:257-262, 1986

2. Bi WL, Smith TR, Nery B, Dunn IF, Laws ER: Pituitary tumors: functioning and nonfunctioning, in Winn HR (ed): Youmans \& Winn Neurological Surgery, ed 7. Philadelphia: Elsevier, 2016, pp 1155-1182

3. Colombo N, Loli P, Vignati F, Scialfa G: MR of corticotropin-secreting pituitary microadenomas. AJNR Am J Neuroradiol 15:1591-1595, 1994

4. Cottier JP, Destrieux C, Vinikoff-Sonier C, Jan M, Herbreteau D: [MRI diagnosis of cavernous sinus invasion by pituitary adenomas.] Ann Endocrinol (Paris) 61:269-274, 2000 (Fr)

5. Dallapiazza R, Bond AE, Grober Y, Louis RG, Payne SC, Oldfield EH, et al: Retrospective analysis of a concurrent series of microscopic versus endoscopic transsphenoidal surgeries for Knosp Grades 0-2 nonfunctioning pituitary macroadenomas at a single institution. J Neurosurg 121:511-517, 2014

6. Dallapiazza RF, Grober Y, Starke RM, Laws ER Jr, Jane JA Jr: Long-term results of endonasal endoscopic transsphenoidal resection of nonfunctioning pituitary macroadenomas. Neurosurgery 76:42-53, 2015

7. Dhandapani S, Singh H, Negm HM, Cohen S, Anand VK, Schwartz TH: Cavernous sinus invasion in pituitary adenomas: systematic review and pooled data meta-analysis of radiologic criteria and comparison of endoscopic and microscopic surgery. World Neurosurg 96:36-46, 2016

8. Dickerman RD, Oldfield EH: Basis of persistent and recurrent Cushing disease: an analysis of findings at repeated pituitary surgery. J Neurosurg 97:1343-1349, 2002

9. Fernandez-Miranda JC, Zwagerman NT, Abhinav K, Lieber S, Wang EW, Snyderman CH, et al: Cavernous sinus compartments from the endoscopic endonasal approach: 
anatomical considerations and surgical relevance to adenoma surgery. J Neurosurg [epub ahead of print September 1, 2017; DOI: 10.3171/2017.2.JNS162214]

10. Frank G, Pasquini E: Endoscopic endonasal cavernous sinus surgery, with special reference to pituitary adenomas. Front Horm Res 34:64-82, 2006

11. Hardy J: Transsphenoidal surgery of hypersecreting pituitary tumors, in Kohler PO, Ross GT (eds): Diagnosis and Treatment of Pituitary Tumors. Amsterdam: Excerpta Medica, 1973, pp 179-198

12. Jane JA Jr, Laws ER Jr: The surgical management of pituitary adenomas in a series of 3,093 patients. J Am Coll Surg 193:651-659, 2001

13. Knappe UJ, Jaspers C, Buschsieweke D, Reinbold WD, Alomari A, Saeger W, et al: Ectopic adrenocorticotropic hormone-secreting pituitary adenomas: an underestimated entity. Neurosurgery 80:525-533, 2017

14. Knosp E, Steiner E, Kitz K, Matula C: Pituitary adenomas with invasion of the cavernous sinus space: a magnetic resonance imaging classification compared with surgical findings. Neurosurgery 33:610-618, 1993

15. Lonser RR, Ksendzovsky A, Wind JJ, Vortmeyer AO, Oldfield $\mathrm{EH}$ : Prospective evaluation of the characteristics and incidence of adenoma-associated dural invasion in Cushing disease. J Neurosurg 116:272-279, 2012

16. Meij BP, Lopes MB, Ellegala DB, Alden TD, Laws ER Jr: The long-term significance of microscopic dural invasion in 354 patients with pituitary adenomas treated with transsphenoidal surgery. J Neurosurg 96:195-208, 2002

17. Molitch ME: Diagnosis and treatment of pituitary adenomas: a review. JAMA 317:516-524, 2017

18. Oldfield EH, Jane JA Jr, Thorner MO, Pledger CL, Sheehan JP, Vance ML: Correlation between GH and IGF-1 during treatment for acromegaly. J Neurosurg 126:1959-1966, 2017

19. Oldfield EH, Vortmeyer AO: Development of a histological pseudocapsule and its use as a surgical capsule in the excision of pituitary tumors. J Neurosurg 104:7-19, 2006

20. Sheehan JM, Vance ML, Sheehan JP, Ellegala DB, Laws ER Jr: Radiosurgery for Cushing's disease after failed transsphenoidal surgery. J Neurosurg 93:738-742, 2000

21. Taylor DG, Jane JA Jr, Oldfield EH: Resection of pituitary macroadenomas via the pseudocapsule along the posterior tumor margin: a cohort study and technical note. J Neurosurg 128:422-428, 2018

\section{Disclosures}

The authors report no conflict of interest concerning the materials or methods used in this study or the findings specified in this paper.

\section{Author Contributions}

Conception and design: Mastorakos, Jane. Acquisition of data: Mastorakos, Taylor. Analysis and interpretation of data: Mastorakos, Taylor, Buell, Donahue. Drafting the article: Chen, Buell. Critically revising the article: Taylor, Chen, Buell, Donahue, Jane. Reviewed submitted version of manuscript: Mastorakos, Taylor, Chen, Jane. Approved the final version of the manuscript on behalf of all authors: Mastorakos. Statistical analysis: Mastorakos, Donahue. Study supervision: Jane.

\section{Correspondence}

John A. Jane Jr.: University of Virginia Health System, Charlottesville, VA.jaj2k@virginia.edu. 